\title{
Mood and Oxytocin Blood Levels in Physically Active Women with and without Oral Contraceptive Use in Relation to Seasonal Day- light Variation
}

\author{
Lisbeth Wikström Frisén ${ }^{1}$, Peter Larsson ${ }^{1}$, Lucia Mincheva Nilsson ${ }^{2}$ and Karin Henriksson Larsén ${ }^{1,3}$ \\ ${ }^{1}$ Department of Community Medicine and Rehabilitation/Sports Medicine Unit, Umeå University, Sweden \\ ${ }^{2}$ Department of Clinical Microbiology/Clinical Immunology, Umeå University, Sweden \\ ${ }^{3}$ The Swedish School of Sport and Health Sciences, Sweden \\ *Corresponding author: Lisbeth Wikström-Frisén, Department of Community Medicine and Rehabilitation/Sports Medicine \\ Unit, Umeå University, SE-901 87 Umeå, Sweden, Tel: +46-90-786-6643, E-mail: lisbeth.wikstrom-frisen@umu.se
}

\begin{abstract}
Background: The aim is to provide normative data on oxytocin, and its seasonality in users and non-users of Oral Contraceptives $(\mathrm{OC})$ in physically active women. Further, to elucidate the relationship between Oxytocin and Profile of Mood States (POMS) for further research to see if Oxytocin can be used as an additional diagnostic marker to detect overreaching/overtraining syndrome or other stress disorders.

Methods: Forty-eight women (18-35 years old), 19 with and 29 without OC use, physically active at least 3 times a week at $>13$ on the Borg RPE scale. Thirty-three subjects completed the study, 15 with and 18 without OC use. The number of hours from sunrise to sunset averaged $10 \mathrm{~h}$ during the autumn, $6 \mathrm{~h}$ during the winter, and $15 \mathrm{~h}$ during the spring. Once a month, blood samples were taken, weight and the day of menstrual cycle was documented, the POMS questionnaire was filled out as well as a personal daily logbook recording exercise type, duration, and intensity. To evaluate seasonal variations in Oxytocin and Global POMS, repeated measures analysis of variance was used.
\end{abstract}

Results: A significant seasonal variation in Oxytocin levels within the groups with and without $O C$ respectively and significant differences in Oxytocin levels between the groups at diverse seasons were found. No significant difference in seasonal variation of Global POMS within the groups and no significant differences in levels of Global POMS between the groups with and without $\mathrm{OC}$ were seen. Furthermore, no clear relationship between Oxytocin, Global POMS scores, and hours of daylight respectively were detected.

Conclusions: Due to seasonality, impact of OC use on oxytocin levels, methodological considerations, and no convincing relationship to Global POMS, oxytocin is not suggested to be an optimal, diagnostic marker alone or in combination with others to detect overreaching and overtraining syndrome in physically active women.

\section{Keywords}

Profile of Mood States (POMS), Oxytocin, Oral Contraceptive (OC), Females, Hormone, Seasonal variation, Daylight

\begin{abstract}
Abbreviations
EDTA: Ethylene Diamine Tetra Acetic acid (EDTA tubes); EIA: Enzyme Immunoassay Assay; ELISA: Enzyme-Linked Immunosorbent Assay; OC: Oral Contraceptive; OD: Optical Density; OT: Oxytocin; POMS: Profile of Mood States; SPSS: Statistical Package for the Social Science
\end{abstract}

\section{Background}

Oxytocin is related to different forms of positive social interactions and stress management [1]. In humans, Oxytocin improves positive communication and reduces stress during conflicts and might be an important central nervous mechanism involved in the stress-protective and have a health-promoting role in positive couple interaction [2] it may also suppress the "classical" stress hormones of the hypothalamic pituitary adrenal axis $[3,4]$. Most of the knowledge about the functions of Oxytocin, however, are results from animal studies [5].

Oxytocin is synthesized in the hypothalamus, transported to the posterior pituitary gland, stored and released into the circulation, acting on target organs.

Citation: Frisén LW, Larsson P, Nilsson LM, Larsén KH (2017) Mood and Oxytocin Blood Levels in Physically Active Women with and without Oral Contraceptive Use in Relation to Seasonal Daylight Variation. Int J Sports Exerc Med 3:058. doi.org/10.23937/2469-5718/1510058

Received: July 15, 2016; Accepted: May 30, 2017; Published: June 01, 2017

Copyright: (C) 2017 Frisén LW, et al. This is an open-access article distributed under the terms of the Creative Commons Attribution License, which permits unrestricted use, distribution, and reproduction in any medium, provided the original author and source are credited. 
Oxytocin is also released within the brain, where it acts at specific oxytocin receptors [6-8]. Studies have also shown that Oxytocin is an omnipresent hormone and synthesized at many sites in the body [6]. Oxytocin and oxytocin receptors expression is usually higher in females than in males, and the central roles of Oxytocin on behaviors and physiology are strongly dependent on steroid hormones and gender. Differences in Oxytocin and oxytocin receptors distributions between brains of the different sexes have been reported [9].

In earlier studies of women not taking oral contraceptives, the secretion of Oxytocin varies over the menstrual cycle, even though only small variations in Oxytocin levels were found [10-13]. Oxytocin concentration has been shown to increase with estradiol administration $[14,15]$ and in pregnancy there is evidence that the magnitude of the changes in circulating concentration is related to the estradiol/progesterone ratio [16]. A significant increase in Oxytocin levels has also been shown when women used oral contraceptives $[17,18]$.

Previous studies have shown that there is a significant circadian and seasonal periodicity in various endocrine functions [19]. Studies have shown that secretion of Oxytocin increased during sleep in humans $[15,20]$ although Nathorst Boos, et al. [14] found minimal diurnal variation of Oxytocin but substantial differences in basic levels between individuals. No studies have so far been performed concerning seasonal periodicity of Oxytocin. Umeå is a town in northern Sweden (located at $63^{\circ} 49^{\prime} 32^{\prime \prime} \mathrm{N} 20^{\circ} 15^{\prime} 49^{\prime \prime} \mathrm{E}$ ) with a large variation in daylight hours during the year. This setting makes studies of seasonal variations in hormones favorable.

Since plasma concentrations and central release of Oxytocin are increased following both physiological, psychological, and social stress [21]. Oxytocin is also thought to work as an Anxiolytic as it decreases stress hormones in both humans and rats, and to modulate the physiological and behavioral responses to stress. Oxytocin also seems to have primarily parasympathetic actions and has been shown to increase directly after endurance running [22] and it facilitates the slowing down of the heart rate in trained individuals [23,24]. Physiological training stress can thus be used as a model to study the relationship between Oxytocin, mood and stress.

Overreaching is defined as an overloading of the body beyond its adaption, the body then needs only a relatively short time for recovery $[25,26]$. The overtraining syndrome, characterized principally by increased fatigue and reduced performance, is described as the result of an excess of training load with an inadequate recovery between training sessions and training periods, combined with psychosocial stress [27-29]. It is assumed that overtraining syndrome is induced when the hypothalamus cannot cope with the total amount of stress, leading to a dysfunction of the neuro endocrine system and changes in behavior and mood [30]. There is a gradual transition from overreaching to overtraining syndrome, and the time needed for recovery is considered to be much longer for overtraining syndrome compared to overreaching [25]. It is therefore important to detect symptoms at an early stage [30].

Overtraining syndrome is accompanied by negative disturbances in the total mood state, determined by the Global Profile of Mood States (Global POMS) and a decreased Vigor/Fatigue ratio [29,30-32] The Profile of Mood States (POMS), an easy-to-use, inexpensive test, originally developed for psychiatric patients as a response test to treatment [33] is also frequently used in athletes to assess affective mood state fluctuation [25,33-35]. Over trained athletes report negatively elevated mood scores and greater perceptual changes in comparison to healthy athletes [32]. In a study by Berglund and Safstrom [31], an elevated Global POMS score $>50 \%$ over the basic level during low season has been used as a criterion for determining whether worldclass canoeists were training excessively or developing staleness. Increased mood disturbances in athletes, or negatively elevated Global POMS, are associated with high training stimulus/stress [30,36].

There are physiological and psychological limits when optimizing physical performance, and hence there is a need for a system to control training, other stressors and recovery [26]. A commonly used marker in men is the Free Testosterone/Cortisol Ratio (FTCR). FTCR is not by itself a suitable indicator for the anabolic/catabolic balance in females, since female free testosterone values are much lower compared to those of men and show a varying response over the season [37]. Thus, no single, specific, simple, and reliable parameter has been shown to diagnose overreaching and overtraining syndrome, and only small improvements have been made in recent years in developing tools suitable for diagnostics, especially for women $[25,38]$. So far impairment of physical performance and disturbed mood profile seem to be the most reliable markers for overtraining [38].

Since Oxytocin is related to mood, further research is needed to elucidate this relationship in healthy and physically active individuals. If such a significant relationship exists, Oxytocin levels in peripheral blood samples might be used as markers to identify physiological and psychosocial stress leading to for example overreaching and overtraining syndrome. Specifically in women, however, there is a need to clarify if the use of oral contraceptives has an impact on these markers and also to determine a possible seasonality.

The aim of the present study is to provide normative data on oxytocin, and its seasonality in users and non-users of oral contraceptives in physically active women. Further to elucidate the relationship between Oxytocin and Profile of Mood States (POMS) for further research to see if Oxytocin can be used as an additional 
Table 1: Subject characteristics, tests availability and basic date of the subject.

\begin{tabular}{|c|c|c|c|c|}
\hline & Inclusion & Monthly & $\begin{array}{l}\text { End of study } \\
\text { OC vs. Non OC }\end{array}$ & $\begin{array}{l}\text { Study Group } \\
\text { OC vs. Non OC }\end{array}$ \\
\hline $\begin{array}{l}\text { Subjects* }^{*} \\
\text { OC vs. Non OC }\end{array}$ & 19 vs. 29 & & $\begin{array}{l}15 \text { (13 combined, } \\
1 \text { antiandrogen, } 1 \text { gestagen), vs. } 18\end{array}$ & \\
\hline Age & $18-35$ yrs & & $23 \pm 3$ yrs vs. $26 \pm 4$ yrs & $p=0.006$ \\
\hline Weight & yes & yes & $61 \pm 6 \mathrm{~kg}$ vs. $67 \pm 13 \mathrm{~kg}$ & $p=0.012$ \\
\hline Height & yes & & $1.66 \pm 0.06 \mathrm{~m}$ vs. $1.68 \pm 0.07 \mathrm{~m}$ & n.s. \\
\hline $\mathbf{V O}_{2 \max }\left(\mathrm{ml} / \mathrm{kg}^{*} \mathrm{~min}\right)$ & $50.0 \pm 4.7$ vs. $46.4 \pm 4.6$ & & $49.9 \pm 5.0$ vs. $45.3 \pm 6.1$ & $\begin{array}{l}\text { Inclusion } p=0.037 \\
\text { End of study } p=0.040\end{array}$ \\
\hline Oxytocin & yes & yes & yes & \\
\hline POMS & yes & yes & Yes & \\
\hline Menstrual cycle day & yes & yes & Yes & \\
\hline Training load $^{* *}$ & $\begin{array}{l}>3 \mathrm{~h} / \mathrm{w} \text { at } \\
>\text { Borg } 13\end{array}$ & yes & $15 \pm 2$ vs. $15 \pm 2$ & n.s. \\
\hline
\end{tabular}

*Reason for becoming a discontinuer: absence $>2$ consecutive tests $(n=5)$, other reasons $(n=4)$, changing OC use $(n=5)$, pregnancy $(n=1)$; "*Borg, 1970; (58) (57), 7 on the training intensity scale in the POMS questionnaire.

diagnostic marker to detect overreaching/overtraining syndrome or other type of stress disorders.

\section{Methods}

\section{Inclusion}

Subjects were recruited by flyers at a local gym and volunteered to take part in the study. Both women with and without Oral Contraceptives (OC) were recruited. Before participation the women were informed of the importance of continuing to be physically active at least three days a week at the same level (Borg RPE scale more than 13) during the study period, and to take at least two days of rest every week to keep a balance between training and recovery to avoid overreaching and overtraining syndrome. A personal daily logbook was given to each subject for recording exercise type, duration, and intensity, to ensure training loads within the given prescription, and note if any health problems occurred. A constant training level was also confirmed by the measurements of the highest oxygen uptake (peak oxygen uptake, $\mathrm{VO}_{2 \text { peak }}$ ) at inclusion and at the end of the study.

All subjects gave written informed consent to participate in the study, and all procedures were approved by The Regional Ethical Review Board in Umeå (Dnr 05 $148 \mathrm{M})$. The subjects were non-smokers and were medically screened to ensure that no medical problem would compromise their participation in the study. Subjects with an irregular menstrual cycle history were excluded from the study. At inclusion the 18 women without OC had menstrual cycles of average 21-35 days. During the study menstrual cycles longer than 35 days (samples, $\mathrm{n}$ $=8$ ) were excluded from the results.

The number of hours from sunrise to sunset in Umeå, provided by the U.S. Naval Observatory Astronomical Applications Department, implicate an average of $10 \mathrm{~h}$ $(6.5 \mathrm{~h}-13 \mathrm{~h})$ during the autumn, $6 \mathrm{~h}(4.5 \mathrm{~h}-8.5 \mathrm{~h})$ during the winter, and $15 \mathrm{~h}(11 \mathrm{~h}-18 \mathrm{~h})$ during the spring.

\section{Study design}

For information on subject inclusion, when the different tests were performed and on basic date of the subject see Table 1.

\section{The Profile of Mood States (POMS)}

In a separate and calm environment the Swedish POMS questionnaire (licensed Data Medic AB \& Melebo AB, copyright EdITS) was filled out. The standard instructions of the POMS questionnaire were used, the subjects answered the question, "How have you been feeling during the past week including today?" [33]. The Profile of Mood States (POMS) test is a 65-item self-report questionnaire which provides an index of total mood disturbance (Global POMS score), and also measures six mood states (tension, depression, anger, fatigue, confusion, and vigor) on a five-point scale from not at all (0) to extremely (4), McNair, et al. [33], adding a constant of 100 to prevent the occurrence of negative score in the Global POMS $[30,31,39]$. To estimate training intensity, a rating scale from 2 (very, very low) to 14 (very, very high) is used to see if a high Global POMS score and a decreased vigor/fatigue ratio is connected to high training intensity.

\section{Aerobic performance}

Aerobic performance was assessed with an incremental test on an electronically braked bicycle (Monark, $839 \mathrm{E}$, Sweden) with a metabolic gas measurement system (MetaMax II, CORTEX, Biophysik GmbH, Leipzig, Germany), a method described in detail in Gilenstam, et al. [40]. For each individual, the cycle ergometer tests were performed identically at the two occasions, at approximately the same time of the day and with the same technical assistance. The highest oxygen uptake $\left(\mathrm{VO}_{2 \text { pe- }}\right.$ $\left.{ }_{\text {ak }}\right) \mathrm{ml} / \mathrm{kg} \times \mathrm{min}$, measured during the incremental cycle ergo meter test was noted.

\section{Blood sample collection}

Blood samples for detection of Oxytocin levels were 
collected once a month, from September to May, giving a total of nine samples for each subject allocated into three groups: autumn (September, October, November), winter (December, January, February), and spring (March, April, May). A solitary missing value for one month was completed with a calculated mean of the existing values from that season, and when values for two months were missing, the existing value was used as the mean for that season. The peripheral blood samples were collected from each subject, with maximal one-week differences between calendar days at different days of the menstrual cycle to represent the whole menstrual cycle. The samples were drawn from an ante cubital vein with the subjects in a seated position in the morning at 07:30 $\mathrm{h}-08: 30 \mathrm{~h}$, about 45 minutes after a light breakfast and without any heavy physical activity or stress before the sampling in order to resemble the ordinary life situation. Samples were collected into chilled EDTA-containing plastic tubes, put on ice directly and immediately centrifuged at $1,600 \times \mathrm{g}$ for 15 minutes at $0{ }^{\circ} \mathrm{C}$. The plasma was then stored at $-80^{\circ} \mathrm{C}$. until laboratory analyses were performed. All samples were analyzed at the same time to assert standardized assay with the same analyze kit and the same calibration.

\section{Oxytocin in plasma}

Oxytocin was analyzed by a competitive EIA methodology with a commercial Oxytocin Enzyme Immunoassay Kit (Assay Designs, Ann Arbor, MI, U.S.A.) according to the manufacturer's instructions. In brief, for the standard curve, 7 two-fold dilutions were prepared from the stock standard corresponding to concentrations ranging from 15.6 to $1,000 \mathrm{pg} / \mathrm{ml}$. One hundred $\mathrm{L}$ of the standard dilutions extracted double plasma samples, and blanks were plated in coated 96 well micro titer plates together with appropriate conjugates and antibodies according to the recommended EIA protocol. The prepared plates were gently tapped to mix, sealed, and incubated for 24 hours at $4{ }^{\circ} \mathrm{C}$. The next day the plates were washed and secondary antibodies, conjugated with alkaline phosphatase and p-nitrophenyl phosphate substrate, were added to each well. The color reaction was allowed to develop for $1 \mathrm{~h}$ at room temperature. Thereafter, a stop solution (trisodium phosphate) was added and the Optical Density (OD) of the color reaction was read immediately in a micro plate reader at $405 \mathrm{~nm}$ wavelength. The Oxytocin concentration $(\mathrm{pg} / \mathrm{ml})$ in the plasma samples was calculated from the standard curve. The lower limits of detection of Oxytocin were 11 $\mathrm{pg} / \mathrm{ml}$, and samples with values below limits of detection were given the lowest value for detection (samples, $n=2$ in women with $\mathrm{OC}$ and samples, $\mathrm{n}=2$ without $\mathrm{OC}$ ). The highest limits of detection were $1,000 \mathrm{pg} / \mathrm{ml}$. Samples with values over the highest limit were given the highest value for detection (samples, $\mathrm{n}=9$ in women with OC and samples, $\mathrm{n}=2$ without $\mathrm{OC}$ ).

\section{Statistical analysis}

Data were analyzed with the Statistical Package for the Social Sciences (SPSS v19). The level of statistical sig- nificance was set at $p<0.05$. The Paired Samples T-Test was used when comparing means within groups, and Independent Samples Test was used when comparing means between groups. Conventional methods were used to calculate the means and Standard Deviations (SD). Statistical analyses of Oxytocin levels and Global POMS scores showed normal distribution in the two study groups with and without $O C$, and no extreme outliers were identified that interfered substantially with the analysis. To evaluate seasonal variations in Oxytocin and Global POMS, repeated measures analysis of variance was used, with season as a within-subject variable and group as a between-subject variable. When the sphericity assumption was violated based on Mauchly's test, the Huynh-Feldt adjustment was used. If there was a significant interaction between season and group, the data were split by the group variable and the seasonal variable, respectively. When data were split by group, a repeated measures analysis of variance with season as a within-subject variable and no between-subject variable was used. If it was found significant, contrast analysis were used to establish which season differed. When data were split by season, the Independent Samples Test was used to compare groups for each season.

Because the Global POMS score, the mood states fatigue and vigor, and the rating of estimated training intensity represent ordinal data, the non-parametric test, Spearman's rank correlation coefficient, was used when analyzing the relationship with Oxytocin and hours of daylight, respectively. Due to related observations with each individual represented multiple times during the study, the p-value could not be calculated for the study group. Instead, correlation analyses were performed for each individual separately, and the numbers of significant correlations in women with and without $\mathrm{OC}$ respectively were summarized. To clarify the correlation between day of the menstrual cycle and the level of Oxytocin, the linear regression was used and $\mathrm{R}^{2}$ was calculated for each individual separately and the numbers of significant correlations in women with and without OC respectively were summarized.

\section{Results}

The two study groups, women with and without OC, were comparable in terms of subject characteristics (Table 1). Mean value and SD of Oxytocin levels (pg/ml) and mean hours of

Table 2: Levels of Oxytocin (OT), Seasonal Daylight Hours and Ratios of Oxytocin Levels in Women with and without Oral Contraceptives (OC).

\begin{tabular}{|l|l|l|l|}
$\begin{array}{l}\text { Mean Seasonal } \\
\text { Daylight Hours }\end{array}$ & $\mathbf{6}$ (Winter) & $\mathbf{1 0}$ (Autumn) & $\mathbf{1 5}$ (Spring) \\
\hline $\begin{array}{l}\text { Oxytocin Level (pg/ml) } \\
\text { Study groups }\end{array}$ & & & \\
\hline Women with OC & $430 \pm 212$ & $370 \pm 215$ & $405 \pm 207$ \\
\hline Women without OC & $301 \pm 149$ & $332 \pm 186$ & $263 \pm 138$ \\
\hline Mean OT Level & & & \\
\hline with OC/without OC & 1.4 & 1.1 & 1.5 \\
\hline
\end{tabular}


daylight from sunrise to sunset during winter, autumn, and spring for women with OC (samples, $n=127$, missing samples $n=8$ ) and without OC (samples, $n=161$, missing samples $n=1$ ) respectively are presented in Table 2 . When analyzing seasonal variation in Oxytocin in the whole study group, a significant interaction between season and group was found ( $p=0.001)$. Separate analysis of women with OC and without OC showed significant seasonal variation in Oxytocin levels for each group separately (with OC, $p=0.027$; without OC, $p=0.003$ ). To establish which season that differed in each group respectively, further statistical analysis was carried out and showed significant differences in women with OC between autumn and winter $(p=0.022)$ and in women without OC between autumn and spring $(p=0.003)$ as well as between winter and spring ( $p$ $=0.032$ ) (Figure 1). Further analysis comparing Oxytocin levels between women with and without $\mathrm{OC}$ in each season showed significantly higher mean levels of Oxytocin in women with OC

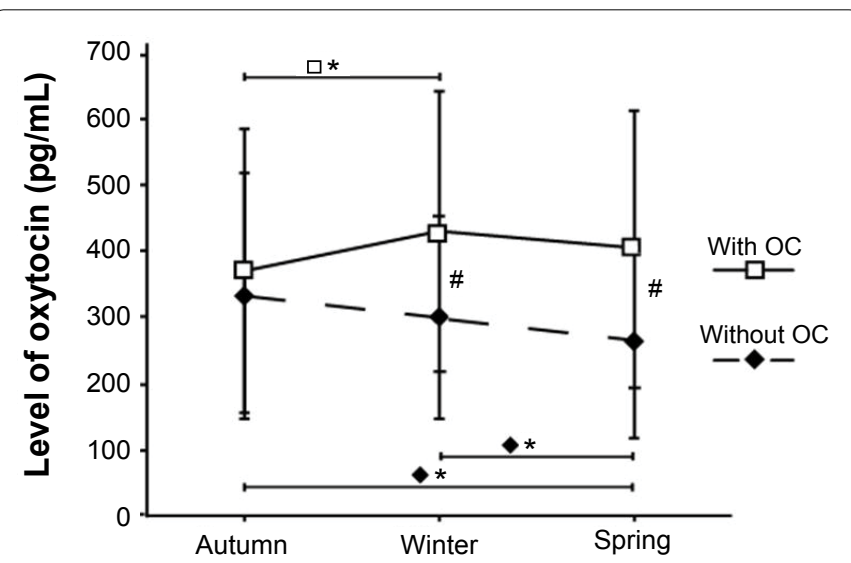

Figure 1: Average concentration of oxytocin levels in women with and without Oral Contraceptives (OC). In autumn, winter, and spring (mean $\pm 1 S D$ ). Statistically significant seasonal variations are shown in women with $\mathrm{OC}\left(\square^{*} p<0.05\right)$ and woman without OC $\left(\star^{\star} p<0.05\right)$. Significant differences in oxytocin levels between the groups are shown $(\# p<0.05)$.

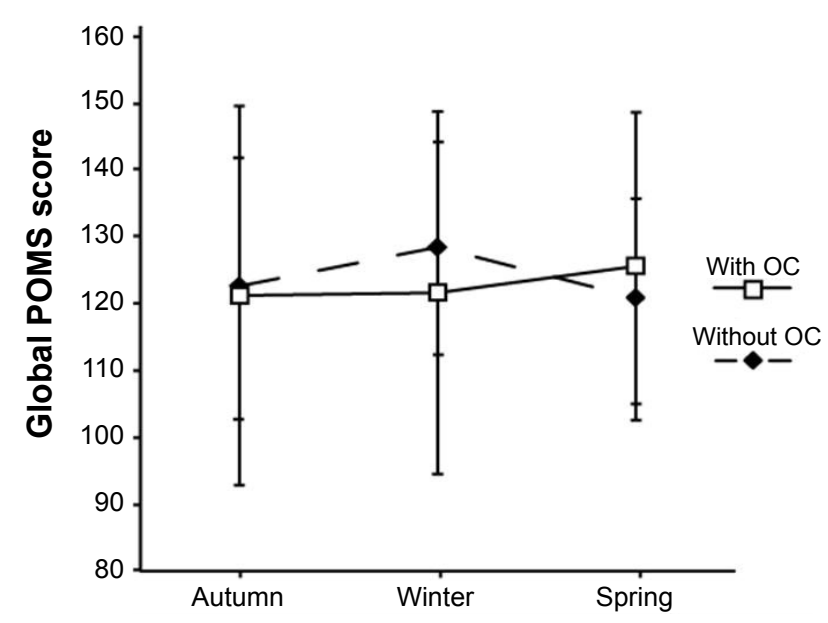

Figure 2: Average Global Profile of Mood States (Global POMS) score in women with and without Oral Contraceptives (OC). In autumn, winter, and spring (mean $\pm 1 \mathrm{SD}$ ). No significant seasonal variation was found in Global POMS score within women with OC (口) and without OC ( $\bullet)$ and no significant differences in levels between the groups were detected. both during the winter $(p=0.048)$ and during the spring ( $p$ $=0.026$ ), but not during the autumn (Figure 1). Women with OC peaked in the winter season $(430 \mathrm{pg} / \mathrm{ml} \pm 212)$, and the lowest mean value was detected during the autumn ( $370 \mathrm{pg} /$ $\mathrm{ml} \pm 215)$. Women without OC peaked during the autumn $(332 \pm 186 \mathrm{pg} / \mathrm{ml})$, and the lowest mean value was detected in the spring season $(263 \mathrm{pg} / \mathrm{ml} \pm 138)$. These results were also reflected by the ratios $>1$ of mean Oxytocin levels with $\mathrm{OC} /$ mean Oxytocin levels without OC (Table 2).

Women with and without OC showed no significant seasonal variation in Global POMS scores within the groups respectively, and no significant differences in levels of Global POMS scores between the groups. The Global POMS score as mean value and standard deviation are presented for the two groups respectively in autumn, winter, and spring (Figure 2). In women with and without $\mathrm{OC}$, the relationship between Oxytocin levels and Global POMS scores could not be calculated for the whole groups due to related samples. The Spearman's correlation coefficient was instead calculated for each woman separately. In this analysis only two women out of 33 women, one with OC and one without OC, displayed a significant relation between Oxytocin levels and Global POMS scores, indicating that there was no tendency for the Global POMS score to either increase or decrease with changes in Oxytocin levels during the study period. When investigating the relationship between Oxytocin levels, Global POMS scores, the six mood states, and estimated training intensity respectively we found a few significant results. Eight women out of 33 (five with OC, 3 without OC) displayed diverse significant relations between Oxytocin and the different mood states. Further, one woman out of 33 (without OC) displayed a significant relation between Oxytocin and estimated training intensity and one woman (without OC) out of 33 displayed a significant relation between Global POMS score and estimated training intensity. Two women out of 33 (without OC) showed a significant relationship between the vigor/fatigue ratios and estimated training intensity.

To clarify the relationship between Oxytocin levels, Global POMS scores, and hours of daylight respectively, each woman was analyzed separately. Only two women out of 33 (one with OC and one without OC) showed a significant relationship between hours of daylight and Oxytocin. When analyzing the relationship between hours of daylight and Global POMS scores, only two women (with OC) out of 33 showed a significant relationship. A significant relationship between day of menstrual cycle and Oxytocin level was found in only three women out of 33, two with OC and one without OC.

\section{Discussion}

The aim of this study was to provide normative data on peripherally measured Oxytocin, to investigate the relationship between Oxytocin and POMS in physically 
active women for further research to see if Oxytocin can be used as an additional diagnostic marker to detect overreaching, overtraining syndrome or other stress disorders. Because significant circadian and seasonal periodicity in various endocrine functions can occur $[15,19,20]$ we found it crucial to determine if that was applicable to Oxytocin levels and to investigate if seasonal variability in daylight hours interacts with Oxytocin, POMS, and their relationship. Measuring seasonal variation in hormonal systems is important for providing a general background and to provide basic data for research in this field. Therefore we measured Oxytocin levels repeatedly over nine months in a location with marked seasonal variability in daylight hours.

Oxytocin can be modulated by ovarian steroids [20] hence to elucidate basic hormonal data in women, the use of $O C$ has to be considered. Our findings indicated seasonal differences in Oxytocin, which showed a different pattern in women using OC compared to those not using OC (Figure 1). Differences in individual Oxytocin levels both in women with and without OC were seen in this study (Table 1), which is supported by Nathorst-Boos, et al. [14], showing substantial differences in basal Oxytocin levels between individuals. Therefore it is probably important to identify individual baseline Oxytocin levels in order to study the individual changes in Oxytocin levels, as in other hormones [41].

Biochemical analysis has been suggested as a way to be able to diagnose training stress and overtraining, but no suitable single test has been identified $[25,28,31,38,42,43]$. Oxytocin has been shown to increase directly after endurance running [22] and seems to have primarily parasympathetic actions such as slowing down the heart rate in trained individuals $[23,24]$ a phenomenon related to training and overtraining syndrome [44]. This study aimed to investigate if analysis of Oxytocin levels could be an additional marker training stress such as overtraining syndrome since Oxytocin has been shown to have effects on stress management [1]. Seasonal periodicities could influence the Oxytocin relation to the Global POMS score and be of importance to control if used as a diagnostic tool to detect signs of overreaching and overtraining syndrome. The results in this study showed seasonal variations and differences in Oxytocin levels between the groups at diverse seasons. This implies that standardizing the time of year for measuring Oxytocin is important both in women with and without $\mathrm{OC}$ when designing studies, analyzing and interpreting results of Oxytocin measurements, and making longer longitudinal studies with Oxytocin difficult.

The present study tried to clarify if extreme seasonal variations in the daylight hours could interact with the level of Oxytocin in women with and without OC living at high latitude. A repeated assessment in this specific location with marked seasonal variability in daylight hours was performed, and the result showed that seasonal variation of Oxytocin probably was not influenced by the number of daylight hours for women with or without OC respectively (Table 2 ). Neither the season with the longest nor the shortest average daylight hours showed a relation too high or low mean Oxytocin levels in the two study groups respectively. Seasonality showed in Oxytocin could be a source of bias in comparative studies in populations not only at higher latitudes.

It is important to optimize performance in life and sports. It is critical to balance between stress, training stress and recovery; this could be the difference between success and failure. In sports psychological and physiological data, POMS scores and blood samples, also training and recovery, as well as performance data are used to estimate the total load to evaluate the state of the individuals, to assess the risk of overreaching and overtraining syndrome. It appears that the total amount of stress exceeding the individual's capacity to cope in combination with a dysfunction of the neuro endocrine system is the reason for developing an overtraining syndrome $[26,27,30,45]$.

The POMS test was used in this study due to its feasibility in sport and exercise studies [33,38]. To identify if Oxytocin, which is related to different forms of positive social interactions and stress management [1] could be a marker of this dysfunction. However, this study could not detect a clear relationship between Oxytocin levels and mood fluctuations, measured as the Global POMS score and the six mood states respectively. This finding is in accordance with a study by Kemp, et al. [46] which showed no change in anxiety, anger, depression, confusion, fatigue, or vigor after administration of Oxytocin intranasal spray.

A study by Cramer, et al. [39] found that 15 weeks of moderate exercise training improved the Global POMS score in sedentary mildly obese women from 120 to 113 raw score while the non-exercisers started and remained at 133 raw score. In this study the training load and the total amount of stress were not heavy enough to cause a severe imbalance in body homeostasis. Suggesting this kind of long term training schedule kept the subjects mood on a constant level. In this study, mean Global POMS scores were found to be between 120 and 130 in both groups during the study period and this could probably be considered to be an average mean level in physically active women and suitable to be compared with Oxytocin levels to provide basic data in women with and without $\mathrm{OC}$. We found no significant difference in seasonal variation of Global POMS scores within the groups and detected no significant differences in levels of Global POMS scores between the groups, but, we detected significant seasonal variations in Oxytocin levels within the groups respectively and significant differences in Oxytocin levels between the groups at diverse seasons. Leading to that no clear relationship between Oxytocin levels, Global POMS scores, and hours of daylight respectively could be detected. 


\section{Methodological Aspects}

The subjects were followed over a long period, September to May, which most likely influenced the dropout rate. This young study population was not settled, and therefore moved, changed their living conditions, and changed contraceptive habits resulting in difficulties with attendance in this longitudinal study. The discontinuers did not differ substantially from the study group. The remaining subjects in the two study groups were comparable in terms of training load during the study period: important to note, as Global POMS was to be evaluated in physically active women with and without OC. A sample was collected once a month for each subject (with a maximal one-week difference) at different days of the menstrual cycle, in order to ensure that various days of the menstrual cycle were covered in the study group. In earlier studies of women not taking OC, the secretion of Oxytocin varied over the menstrual cycle $[11,12]$. Analysis in this study showed only three significant relationships (two women with $O C$ and one without OC) between menstrual cycle day and level of Oxytocin. This indicates that it is unlikely that the menstrual cycle day has an important impact and, therefore cannot explain the differences in Oxytocin levels between women with and without OC in this study.

Although we used an assay with a low respectively high limit of detection, Oxytocin values were below the limit (with OC, samples $n=2$ and without OC, samples $n=2$ ) and over the limit (with OC, samples $n=9$ and without $O C$, samples $n=2$ ), but are unlikely to affect statistical analyses; however supports the results of higher Oxytocin values in women with oral contraceptives [17].

To ensure a correct sampling procedure, blood samples were collected at the same time in the morning, after a light breakfast and without physical activity or stress. A study by Silber, et al. [17] showed no differences in hormonal levels of Oxytocin in plasma taken before and after breakfast, which supports the finding that minimal influence occurred if no breakfast was eaten. We are aware that our Oxytocin levels are somewhat high, but ELISA methodology is known to show higher results compared to RIA. A recent investigation also showed that RIA was generally less sensitive compared to ELISA, giving high numbers of negative or low values in the tested samples, not reflecting the real Oxytocin levels. Thus, there is a critical need for development of more reliable methods for oxytocin measurement [47]. Another possible explanation might be management of the blood samples; and therefore to intend a minimized decomposition process of Oxytocin after the sample collection, a rapid treatment was performed. Blood samples were centrifuged within 5 minutes, and the plasma was immediately frozen and stored at $-80{ }^{\circ} \mathrm{C}$ until ELI$\mathrm{SA}$ assays were performed once directly after thawing. To elucidate the differences in Oxytocin levels between women with and without oral contraceptives, we pres- ent the levels also as ratios of the mean values, which show that physically active women with oral contraceptives have more than 1.1 to 1.5 times higher levels compared to women without oral contraceptives (Table 2). Oxytocin is known to be released into peripheral circulation in a pulsative fashion, as are most of the body's hormones, where it is assumed to have a relatively brief half-life [48] estimated between 1-2 minutes [49] and 3-9 minutes [50] hence it was important to collect the blood sample at the same time; in this study, all blood samples were taken between 07:30 $\mathrm{h}$ and 08:30 $\mathrm{h}$. To provide as comparable and as calm a reception as possible the same routines and technical assistance were used.

Research exploring the role of Oxytocin in human social behavior is difficult because of its release in both body and brain and its interactive effects with other hormones and neuro modulators. Other difficulties are due to the complexity of the blood-brain barrier and oxytocin's instability, which creates measurement issues [51]. Peripheral Oxytocin may or may not reflect centrally released Oxytocin as shown in animal studies [49]. Still, hormone levels are often analyzed in humans with peripheral blood samples [52-56] and for convenience we used this method. Lack of a relationship in this study between peripheral Oxytocin levels and the different POMS scores may be due to differences in peripheral and central secretory patterns in Oxytocin. However, we expect a link between peripheral Oxytocin secretion and mood, a central process, measured by Global POMS. In our study we assumed that plasma Oxytocin concentration correlated with the relevant brain levels of the peptide.

Further studies are needed to examine the relationship between peripheral and central release within the structures where Oxytocin regulates behavior to get a more complete understanding because the research results in the literature on the effects of Oxytocin in the social field are sometimes inconsistent. When improving the quality and quantity of training to optimize performance, the physiological and psychological limits prescribe a need for research that addresses avoiding overreaching and overtraining syndrome. Hence this study tries to evaluate if Global POMS and levels of Oxytocin have a significant correlation in physically active women that can be used in assessment of markers for these states. Future research is warranted on seasonal variation in Oxytocin levels in physically active women with and without $O C$ use because Oxytocin measurements in blood could represent an essential link to interpreting adaptations to physical exercise and recovery. In addition, oxytocin values resulting from this study are highly variable. It might be useful to extend the study to a larger group of people and even change the place of the study, given that there is no significant correlation between the hours of daylight and changes in oxytocin levels, and between the hours of daylight and Global 


\section{POMS scores.}

Oxytocin levels should be interpreted in relation to the levels and responses of other modulators, for example, cortisol. Stress-related health outcomes may depend on the balance between oxytocinergic and fight/ flight for example, cortisol stress responses. Therefore further studies of Oxytocin and its correlation with cortisol should be performed.

\section{Conclusions}

Due to seasonality, impact of OC use on oxytocin levels, methodological considerations, and no convincing relationship to Global POMS, oxytocin is not suggested to be an optimal, diagnostic marker alone or in combination with others to detect overreaching and overtraining syndrome in physically active women. Furthermore, no clear relationship between Oxytocin levels, Global POMS scores, and hours of daylight respectively could be detected. It is important to find a marker to diagnose overtraining syndrome in women, but the oxytocin levels are influenced by too many factors that makes it an unreliable marker.

\section{References}

1. Carter CS, Pournajafi-Nazarloo H, Kramer KM, Ziegler TE, White-Traut R, et al. (2007) Oxytocin: behavioral associations and potential as a salivary biomarker. Ann N Y Acad Sci 1098: 312-322.

2. Ditzen B, Schaer M, Gabriel B, Bodenmann G, Ehlert U, et al. (2009) Intranasal oxytocin increases positive communication and reduces cortisol levels during couple conflict. Biol Psychiatryv 65: 728-731.

3. Heinrichs M, Baumgartner T, Kirschbaum C, Ehlert $U$ (2003) Social support and oxytocin interact to suppress cortisol and subjective responses to psychosocial stress. Biol Psychiatry 54: 1389-1398.

4. Heinrichs M, Von Dawans B, Domes G (2009) Oxytocin, vasopressin and human social behavior. Front Neuroendocrinol 30: 548-557.

5. Gimpl G, Fahrenholz F (2001) The oxytocin receptor system: structure, function, and regulation. Physiol Rev 81: 629-683.

6. Gutkowska J, Jankowski M, Mukaddam-Daher S, McCann SM (2000) Oxytocin is a cardiovascular hormone. Braz J Med Biol Res 33: 625-633.

7. Leng G, Meddle SL, Douglas AJ (2008) Oxytocin and the maternal brain. Curr Opin Pharmacol 8: 731-734.

8. Viero C, Shibuya I, Kitamura N, Verkhratsky A, Fujihara $\mathrm{H}$, et al. (2010) Review: Oxytocin: Crossing the bridge between basic science and pharmacotherapy. CNS Neurosci Ther 16: 138-156.

9. Carter CS (2007) Sex differences in oxytocin and vasopressin: implications for autism spectrum disorders? Behav Brain Res 176: 170-186.

10. Hull ML, Reid RA, Evans JJ, Benny PS, Aickin DR (1995) Pre-ovulatory oxytocin administration promotes the onset of the luteinizing hormone surge in human females. Hum Reprod 10: 2266-2269.

11. Mitchell MD, Haynes PJ, Anderson AB, Turnbull AC (1981)
Plasma oxytocin concentrations during the menstrual cycle. Eur J Obstet Gynecol Reprod Biol 12: 195-200.

12. Steinwall M, Akerlund M, Bossmar T, Forsling ML (1998) Osmotically-induced release of vasopressin and oxytocin in non-pregnant women--influence of estrogen and progesterone. Acta Obstet Gynecol Scand 77: 983-987.

13. Stock S, Bremme K, Uvnäs-Moberg K (1991) Plasma levels of oxytocin during the menstrual cycle, pregnancy and following treatment with HMG. Hum Reprod 6: 1056-1062.

14. Nathorst-Böös J, Stock S, von Schoultz B (1994) Effects of oophorectomy and estrogen treatment on basal levels and 24-h profiles of oxytocin. Gynecol Endocrinol 8: 127-132.

15. Kostoglou-Athanassiou I, Treacher DF, Wheeler MJ, Forsling ML (1998) Melatonin administration and pituitary hormone secretion. Clin Endocrinol (Oxf) 48: 31-37.

16. Fuchs AR, Behrens O, Liu HC (1992) Correlation of nocturnal increase in plasma oxytocin with a decrease in plasma estradiol/progesterone ratio in late pregnancy. Am J Obstet Gynecol 167: 1559-1563.

17. Silber M, Almkvist O, Larsson B, Stock S, Uvnäs-Moberg K (1987) The effect of oral contraceptive pills on levels of oxytocin in plasma and on cognitive functions. Contraception 36: 641-650.

18. Stock S, Karlsson R, von Schoultz B (1994) Serum profiles of oxytocin during oral contraceptive treatment. Gynecol Endocrinol 8: 121-126.

19. Hansen AM, Garde AH, Skovgaard LT, Christensen JM (2001) Seasonal and biological variation of urinary epinephrine, norepinephrine, and cortisol in healthy women. Clin Chim Acta 309: 25-35.

20. Forsling ML (2000) Diurnal rhythms in neurohypophysial function. Exp Physiol 85: 179S-186S.

21. Lee HJ, Macbeth AH, Pagani JH, Young WS 3rd (2009) Oxytocin: the great facilitator of life. Prog Neurobiol 88: 127151.

22. Carter CS (2003) Developmental consequences of oxytocin. Physiol Behav 79: 383-397.

23. Hew-Butler T, Noakes TD, Soldin SJ, Verbalis JG (2008) Acute changes in endocrine and fluid balance markers during high-intensity, steady-state and prolonged endurance running: unexpected increases in oxytocin and brain natriuretic peptide during exercise. Eur J Endocrinol 159: 729-737.

24. Michelini LC (2007) Differential effects of vasopressinergic and oxytocinergic pre-autonomic neurons on circulatory control: reflex mechanisms and changes during exercise. Clin Exp Pharmacol Physiol 34: 369-376.

25. Halson SL, Jeukendrup AE (2004) Does overtraining exist? An analysis of overreaching and overtraining research. Sports Med 34: 967-981.

26. Kenttä G, Hassmén P (1998) Overtraining and recovery. A conceptual model. Sports Med 26: 1-16.

27. Fry RW, Morton AR, Garcia-Webb P, Keast D (1991) Monitoring exercise stress by changes in metabolic and hormonal responses over a 24-h period. Eur J Appl Physiol Occup Physiol 63: 228-234.

28. Kuipers H (1998) Training and overtraining: an introduction. Med Sci Sports Exerc 30: 1137-1139.

29. Lehmann M, Foster C, Keul J (1993) Overtraining in endurance athletes: a brief review. Med Sci Sports Exerc 25: 854-862. 
30. Raglin JS, Morgan WP, O'Connor PJ (1991) Changes in mood states during training in female and male college swimmers. Int J Sports Med 12: 585-589.

31. Berglund B, Säfström H (1994) Psychological monitoring and modulation of training load of world-class canoeists. Med Sci Sports Exerc 26: 1036-1040.

32. Kenttä G, Hassmén P, Raglin JS (2001) Training practices and overtraining syndrome in Swedish age-group athletes. Int J Sports Med 22: 460-465.

33. McNair DM, Lorr M, Dropplemann DM (1971) Profile of Mood States Manual. San Diego: Educational and Industrial Testing Service. Service E and IT, San Diego.

34. Curran SL, Andrykowski M, Studts JL (1995) Short Form of the Profile of Mood States (POMS-SF): Psychometric information. Psychol Assess 7: 80-83.

35. Hassmén P, Blomstrand E (1991) Mood change and marathon running: a pilot study using a Swedish version of the POMS test. Scand J Psychol 32: 225-232.

36. Morgan WP, Brown DR, Raglin JS, O'Connor PJ, Ellickson KA (1987) Psychological monitoring of overtraining and staleness. Br J Sports Med 21: 107-114.

37. Vervoorn C, Vermulst LJ, Boelens-Quist AM, Koppeschaar HP, Erich WB, et al. (1992) Seasonal changes in performance and free testosterone: cortisol ratio of elite female rowers. Eur J Appl Physiol Occup Physiol 64: 14-21.

38. Ackel-D'Elia C, Vancini RL, Castelo A, Nouailhetas VL, Silva AC (2010) Absence of the predisposing factors and signs and symptoms usually associated with overreaching and overtraining in physical fitness centers. Clinics (Sao Paulo) 65: 1161-1166

39. Cramer SR, Nieman DC, Lee JW (1991) The effects of moderate exercise training on psychological well-being and mood state in women. J Psychosom Res 35: 437-449.

40. Gilenstam KM, Thorsen K, Henriksson-Larsén KB (2011) Physiological correlates of skating performance in women's and men's ice hockey. J Strength Cond Res 25: 2133-2142.

41. Uusitalo AL, Huttunen P, Hanin Y, Uusitalo AJ, Rusko HK (1998) Hormonal responses to endurance training and overtraining in female athletes. Clin J Sport Med 8: 178-186.

42. Urhausen A, Gabriel H, Kindermann W (1995) Blood hormones as markers of training stress and overtraining. Sports Med 20: 251-276.

43. Urhausen A, Kindermann W (2002) Diagnosis of overtraining: what tools do we have? Sports Med 32: 95-102.
44. Hedelin R, Kenttä G, Wiklund U, Bjerle P, Henriksson-Larsén K (2000) Short-term overtraining: effects on performance, circulatory responses, and heart rate variability. Med Sci Sports Exerc 32: 1480-1484.

45. Kellmann M (2010) Preventing overtraining in athletes in high-intensity sports and stress/recovery monitoring. Scand J Med Sci Sports 20: 95-102.

46. Fry RW, Morton AR, Keast D (1991) Overtraining in athletes. An update. Sports Med 12: 32-65.

47. Kemp AH, Quintana DS, Kuhnert R-L, Griffiths K, Hickie IB, et al. (2012) Oxytocin Increases Heart Rate Variability in Humans at Rest: Implications for Social Approach-Related Motivation and Capacity for Social Engagement. PLoS One 7: e44014.

48. Szeto A, McCabe PM, Nation DA, Tabak BA, Rossetti MA, et al. (2011) Evaluation of enzyme immunoassay and radioimmunoassay methods for the measurement of plasma oxytocin. Psychosom Med 73: 393-400.

49. Amico JA, Ulbrecht JS, Robinson AG (1987) Clearance studies of oxytocin in humans using radioimmunoassay measurements of the hormone in plasma and urine. J Clin Endocrinol Metab 64: 340-345.

50. Veening JG, de Jong T, Barendregt HP (2010) Oxytocin-messages via the cerebrospinal fluid: behavioral effects; a review. Physiol Behav 101: 193-210.

51. Churchland PS, Winkielman P (2012) Modulating social behavior with oxytocin: how does it work? What does it mean? Horm Behav 61: 392-399.

52. Guastella AJ, Howard AL, Dadds MR, Mitchell P, Carson DS (2009) A randomized controlled trial of intranasal oxytocin as an adjunct to exposure therapy for social anxiety disorder. Psychoneuroendocrinology 34: 917-923.

53. Onaka T, Takayanagi Y, Yoshida M (2012) Roles of oxytocin neurones in the control of stress, energy metabolism, and social behaviour. J Neuroendocrinol 24: 587-598.

54. Cardoso C, Ellenbogen MA, Orlando MA, Bacon SL, Joober R (2013) Intranasal oxytocin attenuates the cortisol response to physical stress: a dose-response study. Psychoneuroendocrinology 38: 399-407.

55. Borg G (1970) Perceived exertion as an indicator of somatic stress. Scand J Rehabil Med 2: 92-98.

56. Borg G, Hassmén P, Lagerström M (1987) Perceived exertion related to heart rate and blood lactate during arm and leg exercise. Eur J Appl Physiol Occup Physiol 56: 679-685. 